\title{
Assessment of Serum Fatty Acid Binding Protein 4 in Patients with Vitilgo
}

\author{
A.A.Ibrahim, A.I.Mustafa, O.S.El-Shimi and M.A.Metwally
}

Dermatology, Venereology and Andrology Dept., Faculty of Medicine, Benha Univ., Benha, Egypt

E-Mail:Muhammad@gmail.com

\begin{abstract}
Although FABP4 is the most commonly known isoform of FABP, no studies have investigated its role in vitiligo. Therefore, our aim was to assess serum FABP4 level in patients with vitiligo. This is a prospective case-control study and was include50 patients suffering from vitilgo. In addition, 30 apparently healthy individuals. Estimation of serum level of FABP4 using Enzyme Linked Immunosorbent assay (ELISA). Serum FABP4 was significantly higher in vitiligo patients when compared to control group $(p<0.001)$.Higher FABP4 level was significantly associated with overweight. FABP4 showed significant positive correlation with BMI, TC, TG, LDL VASI score, significant negative correlations with HDL in cases. FABP4 could be used to predict metabolic syndrome and cardiovascular disease development in vitiligo patients.. In addition, lipid profile, BMI and VASI of the vitiligo are significantly correlated with FABP4.
\end{abstract}

\section{Introduction}

Vitiligo is a procured, on-infectious malady with a rate of 60 people for each 100000 every year portrayed by dynamic inconsistent, multifocal depigmentation of skin, with or without depigmentation of overlying hair, and mucous films results from loss of melanocytes from the included zones. In spite of the fact that noninfectious, the phenotype of this issue has high effect on the mental conduct of the influenced people than their physical capacity; all the more regularly lead to social separation [1].

Etiological speculations recommended for etiopathogenesis of vitiligo incorporate hereditary, immunological, neuro-hormonal, cytotoxic, oxidative pressure and melanocytorrhagy. Qualities conceivably play arole in all parts of pathogenesis of vitiligo. Most qualities related with weakness to vitiligo are associated with insusceptible guideline and invulnerable focusing of melanocytes. The immune system and hereditary speculation was at first upheld by bunching of vitiligo cases in specific families (Multiplex vitiligo families) and expanded recurrence of immune system sicknesses in patients of vitiligo as in their first degree family members (with or without vitiligo) all the more so in multiplex vitiligo families [2].

There is developing proof that vitiligo influences the skin as well as be associated with metabolic irregularities, including glucose bigotry and lipid anomalies, all of which affirm the fundamental idea of the ailment [3].

Lipids, for example, unsaturated fats (FA) and their subsidiaries, perform different organic capacities, remembering their part for vitality homeostasis, the arrangement of substrates for cell films, and flagging particles in incendiary and metabolic pathways [4]. Unsettling influences in lipid homeostasis can prompt various regular issues including corpulence, insulin obstruction (IR), diabetes mellitus (DM), cardiovascular illness, and even skin issues [5].

Unsaturated fats, which comprise about $15 \%$ of lipids in the layer corneum, take an interest in keeping up the penetrability of the epidermis, for instance, by animating fermentation of the horny layer. Exploration has indicated that deviations in FA sythesis in keratinocytes might be engaged with the pathogenesis of provocative dermatosis, for example, atopic dermatitis or psoriasis. FA moved to long-chain unsaturated fats (LCFA) are moved by specific proteins to different tissues, where they are processed, put away or used [4].

Adipocyte unsaturated fat restricting protein (FABP4), additionally named adipocyte protein 2 (aP2), is an adipokine orchestrated and delivered dominatingly from adipocytes and, less significantly, created in macrophages and endothelial cells [6].

FABP4, with a sub-atomic mass of $14.6 \mathrm{kDa}$ and 132 amino acids, represents up to $6 \%$ of complete cell proteins. The quality encoding this adipokine is situated on chromosome 8q21. Raised degrees of FABP4 are firmly connected with the advancement of stoutness, IR, DM, hypertension and atherosclerosis [7].

The point of the current examination was to survey serum FABP4 level in patients with vitilgo.

\section{Patient and method}

This is a planned case-control study and was include50 patients experiencing vitilgo. What's more, 30 obviously solid people will be picked as a benchmark group. All patients were chosen from the outpatient facility of Dermatology and Andrology Department of Benha University Hospitals.

The examination was affirmed by the morals panel on research including human subjects of Benha Faculty of Medicine. Educated assents will be gotten from all members.

\section{Incorporation rules}

- Patients determined to have vitiligo clinically and analysis will be affirmed with dermoscopy.

- Patients with various clinical varities of vitiligo.

- Patients $\geq 18$ years.

- Both sexual orientations.

Avoidance measures

- Patients under any dietary limitations.

- Patients with dyslipidemia.

- Obese patients.

- Patients with provocative issues as incendiary inside ailment.

All patients will be exposed to the accompanying:- 


\section{Full clinical history}

Complete history will be taken from every patient including individual, present history and history of medication consumption.

\section{Complete clinical assessment}

Complete general and dermatological clinical assessment will be finished. Clinical subtleties of all patients will be recorded.

Skin break out patients will be separated by BMI to four gatherings (WHO, 1995):

- Underweight: under 18.5.

- Normal weight: 18.5 to 25 .

- Overweight: 25 to 30.

- Obese: more than 30.

\section{Evaluation of FABP4 serum level}

Assessment of serum level of FABP4 utilizing

Enzyme Linked Immunosorbent examine (ELISA) .

Appraisal of lipid profile.

\section{Measurable methods}

The gathered information was modified, coded, arranged and acquainted with a PC utilizing Statistical bundle for Social Science (IBM Corp. Delivered 2011. IBM SPSS Statistics for Windows, Version 20.0. Armonk, NY: IBM Corp.). Information were introduced and appropriate investigation was finished by the sort of information got for each parameter.

\section{Results}

Our results showed a comparison of age and gender between all studied groups. The mean age of vitiligo patients was 32.7 years. Male patients represented $62 \%$, while females represented $38 \%$ in vitiligo group. In addition to 30 healthy control subjects of matched age and gender.

Patients and control subjects were age and sex matched ( $p>0.05)$.

BMI and family history did not differ significantly between both groups $(\mathrm{p}=0.704,0.954$ and 0.336 respectively).

Table (1) Comparison of lipid profile data between all studied groups.

\begin{tabular}{lccccc}
\hline & \multicolumn{2}{c}{ vitiligo } & \multicolumn{2}{c}{ Control } & \multirow{2}{*}{ N=30 } \\
\cline { 2 - 5 } & mean & SD & mean & SD & \\
\hline TC (mg/dL) & 197.4 & 43.4 & 173.7 & 23.7 & 0.007 \\
TG (mg/dL) & 137.4 & 39.6 & 114.6 & 17.5 & 0.004 \\
HDL (mg/dL) & 51.5 & 6.2 & 57.3 & 4.6 & 0.001 \\
LDL (mg/dL) & 118.4 & 31.2 & 95.8 & 26.9 & 0.009 \\
\hline
\end{tabular}

Numerical data are expressed as mean, $\mathrm{SD}$, looked at utilizing $\mathrm{t}$ test.

Serum TC, TG, LDL-C indicated essentially more elevated levels while HDL demonstrated fundamentally lower focus in vitiligo patients when contrasted with control gatherings $(\mathrm{p}=0.007,0.004,0.009$ and 0.001 individually).

Mean sickness length was 4.8 years,; middle VASI score was 2 , gone from 0.1 to $94 ; 18 \%$ endured of fixed, while $82 \%$ had dynamic course.

Face was influenced in half, neck in $26 \%$, trunk in $44 \%$, limits in $72 \%$ of every contemplated case. Summed up type was available in $62 \%$, central in $14 \%$, Acrofacial in $14 \%$, segmental in $6 \%$, widespread in $5 \%$.

The vast majority of contemplated patients got treatment $(84 \%)$ and just $16 \%$ didn't; $62 \%$ had skin treatment, $36 \%$ had UVB and 54\% had gotten cancer prevention agent.

Serum FABP4 was altogether higher in vitiligo patients when contrasted with control gathering $(\mathrm{p}<0.001)$.

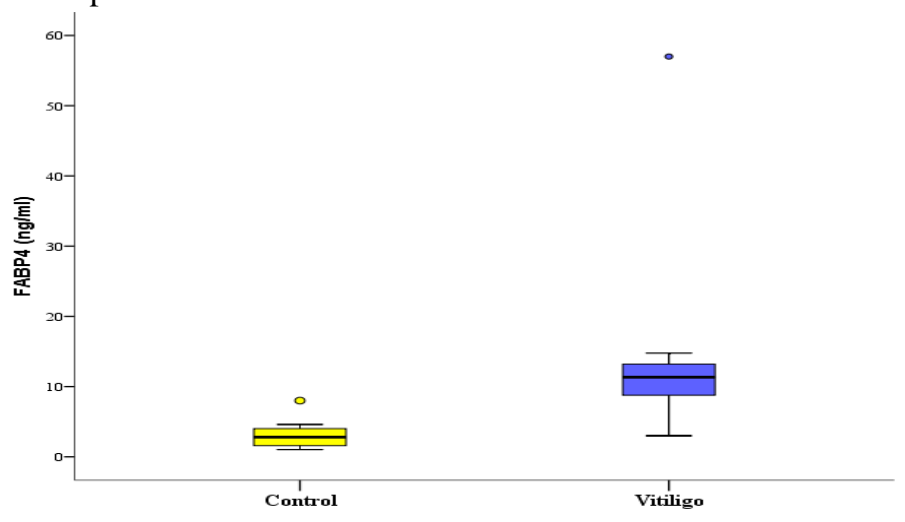

Fig (1) Comparison of serum FABP4 between all studied groups. 
Receiver operating characteristic (ROC) curve of serum FABP4 was conducted for discrimination between vitiligo and control groups. Excellent AUC was found
(AUC $=0.985, \mathrm{p}<0.001)$, Performance criteria at best cut of value are shown in table (1).

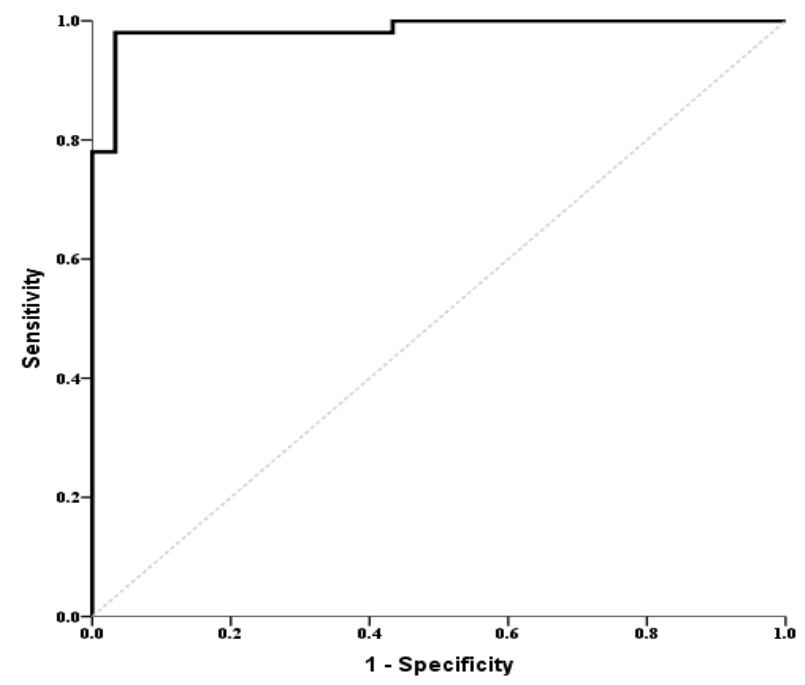

Fig (2) ROC curve of FABP4 for discrimination between vitiligo and control groups.

FABP4 showed significant positive correlation with BMI, TC, TG, LDL VASI score, significant negative correlations with HDL in cases. Otherwise, no significant correlations were found between FABP4 with other parameters in all studied cases.

\section{Discussion}

Vitiligo is one of the skin ailments that cause white spots because of loss of skin shade cells, which is considered as an immune system malady [8].

The commonness of vitiligo is different somewhere in the range of $0.38 \%$ and $2.9 \%$ in worldwide that influence all races and two sexual orientations. Other immune system issues upgrade the commonness of this illness. The specific etiopathogenic instrument of vitiligo isn't perceived. Loss of melanocytes might be related with immune system cytotoxic T-cells, oxidant-cancer prevention agent irregularity, hereditary variables, neural components, or multifactorial instruments. Cell autoimmunity assumes a significant part in vitiligo pathogenesis [9].

Elements of safe framework might be impacted by the dietary status; lipids as essential segments in slims down have significant impact on the tweak of the resistant framework. It has been shown in the various test contemplates. A few investigations uncovered the significance of unsaturated fats in the eating regimen and their application on the decrease of normal symptomatologies in immune system infections [10].

Adipocyte unsaturated fat protein (A-FABP), known as FABP4, is one of the proteins found in develop adipocytes. It has a place with the FABP family and comprises of intracellular lipid transporters that take an interest in directing lipid transport and digestion. FABP4 is discharged from adipocytes and macrophages and has as of late been researched as a marker that is firmly connected with weight and metabolic condition [11].
Despite the fact that FABP4 is the most normally known isoform of FABP, no examinations have explored its function in vitiligo. Hence, our point was to survey serum FABP4 level in patients with vitiligo.

S.M.Derakhshandeh-Rishehri and M.Sallam et al., $[9,12]$ revealed a comparable outcomes to our own that we found no huge contrasts between the cases and the controls regarding age, and sex. Then, A.S.Karadag and M.Rodríguez-Martín et al., [13,8] detailed no critical contrasts could be seen in BMI and this in concurrence with our discoveries. In any case, M.Sallam et al., [12] found that the patient gathering indicated essentially lower BMI than the benchmark group. This might be clarified by that [12] patients and controls have comparable fasting glucose and that patients with vitiligo would in general present lower CVD hazard than controls.

In our examination, we found that Serum TC, TG, LDL-C levels were essentially higher while HDL was fundamentally lower in vitiligo patients contrasted with control gatherings. In steadiness, [13] revealed that vitiligo patients had fundamentally lower HDL-C levels than controls.

Our discoveries were in conflict with M.Sallam et al., [12] and M.Rodríguez-Martín et al., [8] who found a lower noteworthy pervasiveness of changed WC, TGs, and low HDL-C in vitiligo patients. This might be clarify that patients with vitiligo would in general present lower CVD hazard than controls, as vitiligo patients introduced a superior lipid profile, with more elevated levels of HDL-C and lower TGs and WC values.

M.Rodríguez-Martín et al., [8] clarified that these outcomes were a consequence of expanded degrees of superoxide dismutase and glutathione peroxidase in patients with dynamic vitiligo. This upregulation could be an enzymatic premise that makes vitiligo patients less inclined to CVD. Nonetheless, this clarification is 
questioned, as oxidative pressure presents in vitiligo and it is liable for the cytotoxic consequences for melanocytes. Overabundance responsive oxygen species (ROS) were recorded in dynamic vitiligo skin - for instance, high $\mathrm{H} 2 \mathrm{O} 2$ levels were found all through the epidermis [14].

Nonetheless, A.S.Karadag et al., [13] detailed that no huge contrasts were available between vitiligo patients and controls with respect to fasting blood glucose, serum TGs, and diastolic circulatory strain estimations. These progressions could be because of IR and hyperinsulinemia found in vitiligo patients. Hyperinsulinemia may build the creation of lowthickness lipoprotein TGs, and IR can raise circulatory strain [15].

Then again, examination of lipid aggravations in vitiligo kids uncovered diminished degrees of HDL-C just as expanded TGs focuses in the contemplated gathering of vitiligo young ladies [16]. In another investigation by [16], HDL-C fixation was altogether lower in the examined vitiligo youngsters in examination with the solid benchmark group.

Furthermore, A.Pietrzak et al., [16] detailed that HDL-C focus was altogether lower in youngsters with vitiligo contrasted and solid controls, while no critical contrasts were watched with respect to tallness, weight, BMI, fasting serum glucose, and serum TGs of kids with vitiligo and sound kids.

This can be clarified as proinflammatory cytokines TNF, IL-1, IL-6 and other provocative variables (for example C-receptive protein) are associated with summoning IR just as other metabolic intricacies and atherosclerosis 8. Likewise, more elevated levels of homocysteine detailed in vitiligo patients might be engaged with the improvement of the metabolic unsettling influences in vitiligo patients and increment CVD hazard [13, 18].

Exorbitant ROS is known to be liable for lipid peroxidation, protein oxidation, and oxidative DNA harm [18]. The cycles advancing lipid peroxidation that happen in the epidermis, and maybe in the fat tissue also, might be a conceivable clarification for the lipid irregularities recognized [16].

In our investigation, FABP4 was discovered to be essentially higher in vitiligo patients when contrasted with control gathering. Moreover, age, lipid profile, BMI and VASI of the vitiligo patients indicated noteworthy positive relationships with FABP4.

A few investigations have noted positive relationships among's FABP4 and high-thickness lipoprotein (HDL) and low-thickness lipoprotein (LDL) cholesterol, triacylglycerol (TAG) and BMI [19, 20, 21].

Notwithstanding, [22] study on psoriasis patients uncovered no measurably critical relationships with FABP were noted for any of the explored lipid boundaries, either for glucose levels or BMI.

A.Baran et al., [22] revealed that serum FABP4 levels were fundamentally expanded in patients with psoriasis, demonstrating that this protein might be a marker of psoriasis and an autonomous indicator of the danger of comorbidities or intricacies in psoriatic patients.

FABP4 is discharged by fat cells and the blood fixation is identified with corpulence, diabetes, and arteriosclerosis [23].

A past report, [24] detailed that raised degrees of FABP4 added to height of pulse and an atherogenic metabolic phenotype. FABP4 fixation is related with carotid atherosclerosis. FABP4 is believed to be a marker of pathophysiology and could be an objective of treatment in T2DM [25].

In fat mice, FABP4 erasure improved insulin affectability and lipid metabolic issues [26]. Raised serum levels of FABP4 were related with stoutness, insulin obstruction, dyslipidemia and hypertension in solid individuals [27].

Another investigation focusing on non-DM subjects, uncovered that serum FABP fixation were contrarily related with the mean pace of glucose mixture for the last $30 \mathrm{~min}$ of the cinch test utilized as a list of insulin affectability [24].

FABP4-lacking mice demonstrated an expansion in body weight yet decreased insulin obstruction in both high-fat eating routine incited and hereditary stoutness mouse models [28]. Indeed, FABP4-inadequate adipocytes displayed diminished productivity of lipolysis. Knockdown of Fabp4 quality by RNA obstruction in dietary large mice expanded body weight and fat mass without huge changes in glucose and lipid homeostasis [29].

Articulation of FABP4 is prompted during separation from monocytes to macrophages and by treatment with lipopolysaccharide (LPS), phorbol 12myristate 13-acetic acid derivation, PPAR $\gamma$ agonists, oxidized low-thickness lipoprotein, and progressed glycation finished results [30]. On the other hand, atorvastatin, a cholesterol-bringing down medication, has been appeared to lessen FABP4 articulation in macrophages. Metformin, an antidiabetic drug, has likewise been accounted for to hinder forkhead box protein $\mathrm{O} 1$ (FOXO1)- interceded record of FABP4 and lessen lipid amassing in macrophages [31].

\section{References}

[1] A.Alkhateeb, P.R.Fain, A.Thody. Epidemiology of vitiligo and associated autoimmune diseases in Caucasian probands and their families. Pigment Cell Res, Vol.5, PP. 16:208, 2003.

[2] G.F.Mohammed, A.H.Gomaa, MS.Al-Dhubaibi. Highlights in pathogenesis of vitiligo. World J Clin Cases.vol 3,pp221,2015.

[3] A.Pietrzak, J.Bartosinska, J.Hercogova, T.M .Lotti, G.Chodorowska. Metabolic syndrome in vitiligo. Dermatol Ther.vol 25, PP.41-43,2012.

[4] A. J.Smith, B. R.Thompson, M.A.Sanders, D. A. Bernlohr. Interaction of the adipocyte fatty acidbinding protein with the hormone-sensitive lipase: regulation by fatty acids and phosphorylation. J. Biol. Chem, Vol.282, PP.32424-32432,2007.

[5] M.Furuhashi, G.Tuncman, C.Z.Görgün. 
Vaillancourt,Treatment of diabetes and atherosclerosis by inhibiting fatty-acid-binding protein aP2. Nature. June 21, Vol.447(7147), PP.959-65. 2007.

[6] S.Kralisch, M.Fasshauer. Adiocyte fatty acid binding protein: a novel adipokine involved in pathogenesis of metabolic and vascular disease? Diabetologia,vol .56 (1), PP.10-21, 2013.

[7] M.Furuhashi, S.Saitoh, K.Shimamoto, T.Miura. Fatty acid-binding protein 4 (FABP4): pathophysiological insights and potent clinical biomarker of metabolic and cardiovascular diseases. Clin Med Insights Cardiol, Vol.8(Suppl 3), PP.2333, 2015.

[8] M.Rodríguez-Martín, M.Sáez, N.Merino. When are laboratory tests indicated in patients with vitiligo? Dermatoendocrinol.vol 4, PP.53-7,2012.

[9] S.M.Derakhshandeh-Rishehri, M.Heidari-Beni, F. Jaffary. Role of Fatty Acids Intake in Generalized Vitiligo. Int J Prev Med.vol .10,pol .52,2019.

[10] J.E.Löfvenborg, T.Andersson, P.O.Carlsson. Fatty fish consumption and risk of latent autoimmune diabetes in adults. Nutr Diabetes, Vol.4, PP.e139,2014.

[11] S.Ishimura, M.Furuhashi, Y.Watanabe. Circulating levels of fatty acid-binding protein family and metabolic phenotype in the general population. PLoS One.vol .8(11), Pp.e81318, 2013.

[12] M.Sallam, M.A .Gaballah, A.F.State, M.Al-Harrass. Metabolic syndrome in Egyptian patients with vitiligo: a case-control study. J Egypt Women Dermatol Soc, Vol.14,pol. 100-105,2017.

[13] A.S.Karadag, E.Tutal, D.T.Ertugrul.Serum holotranscobalamine. vitamin B12, folic acid and homocysteine levels in patients with vitiligo. Clin Exp Dermatol, Vol.37, PP.62-64,2012.

[14] M.Arunachalam, F.Dragoni, R.Colucci . Nonsegmental vitiligo and psoriasis comorbidity - a case-control study in Italian patients. J Eur Acad Dermatol Venereol, Vol. 28, PP.433-437,2014.

[15] Grundy S.Obesity, metabolic syndrome, cardiovascular disease. J Clin Endocrinol Metab, Vol. 89, PP.2595-2600,2004.

[16] A.Pietrzak, J.Bartosinska, E.Dybiec. Hepato-splenic and lipid profile abnormalities - do they exist in children affected with vitiligo? Acta Dermatovenerol Croat, Vol.22, PP.19-25, 2014.

[17] J.I.Silverberg,NB.Silverberg. Serum homocysteine as a biomarker of vitiligo vulgaris severity: a pilot study. J Am Acad Dermatol, Vol. 64, PP.445447,2011.

[18] S.S. Zhou, D.Li, YM. Zhou, J.M. Cao. The skin function:a factor of anti-metabolic syndrome. Diabetol Metab Syndr, Vol.4, PP.15,2012.

[19]A.Xu, A.W.Tso, B.M.Cheung. Circulating adipocyte-fatty acid binding protein levels predict the development of the metabolic syndrome: a 5year prospective study. Circulation, Vol.115,
PP.1537-1543,2007.

[20] S.Kralisch, M.Fasshauer. Adiocyte fatty acid binding protein: a novel adipokine involved in pathogenesis of metabolic and vascular disease? Diabetologia, Vol.56(1), PP.10-21. doi: 10.1007/s00125-012-2737-4,2013.

[21] CL. Huang, Y.W.Wu, C.C. Wu. Association between serum adipocyte fatty-acid binding protein concentrations, left ventricular function and myocardial perfusion abnormalities in patients with coronary artery disease. Cardiovasc Diabetol, Vol.12, PP.105. , 2013.

[22] A.Baran, M.Świderska, J.Bacharewicz-Szczerbicka. Serum Fatty Acid-Binding Protein 4 is Increased in Patients with Psoriasis. Lipids, Vol.52(1), PP.5160,2017.

[23] A.Xu, Y.Wang, J.Y. Xu. Adipocyte fatty acidbinding protein is a plasma biomarker closely associated with obesity and metabolic syndrome. Clin Chem, Vol.52(3), PP.405-13,2006.

[24]H.Ota, M.Furuhashi, S.Ishimura. Elevation of fatty acid-binding protein 4 is predisposed by family history of hypertension and contributes to blood pressure elevation. Am J Hypertens, Vol.25(10), PP.1124-30,2012.

[25] R. Nakamura, T.Okura, Y.Fujioka. Serum fatty acidbinding protein 4 (FABP4) concentration is associated with insulin resistance in peripheral tissues, A clinical study. PLoS One, Vol.12(6), PP.e0179737,2017.

[26] M .Furuhashi G.S. Hotamisligil. Fatty acid-binding proteins: role in metabolic diseases and potential as drug targets. Nat. Rev. Drug Discov, Vol. 7, PP.489-503, 2008.

[27] S.Zhang, L.Yang, P.Chen. Circulating Adipocyte Fatty Acid Binding Protein (FABP4) Levels Are Associated with Irisin in the Middle-Aged General Chinese Population. PLoS One. January 11, Vol.11(1), PP.e0146605,2016.

[28] K.T.Uysal, L .Scheja, SM.Wiesbrock, Bonner-Weir S, Hotamisligil GS. Improved glucose and lipid metabolism in genetically obese mice lacking aP2. Endocrinology, Vol.141(9), PP.3388-96,2000.

[29]R.Yang, G.Castriota, Y.Chen,. RNAi-mediated germline knockdown of FABP4 increases body weight but does not improve the deranged nutrient metabolism of diet-induced obese mice. Int J Obes, Vol.35(2), PP.217-25,2011.

[30]X.Q.Wang, K.Yang, Y.S.He, L.Lu. Receptor mediated elevation in FABP4 levels by advanced glycation end products induces cholesterol and triacylglycerol accumulation in THP-1 macrophages. Lipids, Vol.46(6), PP.479-86,2011.

[31]J.Song, P.Ren, L.Zhang. Metformin reduces lipid accumulation in macrophages by inhibiting FOXO1mediated transcription of fatty acid-binding protein 4. Biochem Biophys Res Commun, Vol.393(1), PP.89-94,2010. 